\title{
Rare Concomitant Myxoid and Cystic Degeneration of Uterine Leiomyoma - Case Report
}

Igor Samardjiski, ${ }^{1}$ Gordana Petrushevska, ${ }^{2}$ Slagjana Simeonova Krstevska, Iva Paneva, ${ }^{1}$ Vesna Livrinova, ${ }^{1}$ Irena Todorovska, ${ }^{1}$ Maja Pejkovska llieva, ${ }^{1}$ Sasho Dimitrovski, ${ }^{1}$ Katerina Nikoloska ${ }^{1}$

\begin{abstract}
The uterine leiomyomas are monoclonal tumours of myometrial smooth muscle cells that are oestrogen dependent. A 43-year-old patient was referred by her gynaecologist under a suspected diagnosis of ovarian tumour. She complained of prolonged and profuse, regular menstrual bleeding that leaded to anaemia. The ultrasound examination showed a complex tumour mass with dimensions 68 × $85 \mathrm{~mm}$, with several cystic formations fulfilled with clear fluid, which protrudes from the posterior wall of the uterus. Therefore, the diagnosis of leiomyoma with a cystic degeneration was considered preoperatively. In the case of cystic degeneration of the myoma, the ultrasound shows a combination of cystic and solid components with irregular shape and variable echogenicity. The ovarian malignancy should be ruled out in the presence of a large cystic mass with irregular septa and solid nodular parts filling the small pelvis. Ultrasound characteristics of degenerative myomas should always be considered, so as not to replace it with an ovarian mass, especially if it is subserosal and on the loop.
\end{abstract}

Key words: Leiomyoma; Myxoid degeneration; Cystic degeneration; Ovarian tumour; Differential diagnosis.
(1) University Clinic of Obstetrics and Gynaecology, Skopje, North Macedonia.

(2) Institute of Pathological Anatomy, Skopje, North Macedonia.

Correspondence:

IGOR SAMARDJISKI

E: igidoc@yahoo.com

ARTICLE INFO

Received: 22 March 2021

Revision received: 24 August 2021 Accepted: 25 August 2021

\section{Introduction}

The uterine leiomyoma is the most common pelvic tumour in the female population. The exact incidence is difficult to be determine as all the studies are either based on symptomatic cases or histopathological evaluation of the preparations after hysterectomy. Nevertheless, the leiomyomas are thought to be clinically diagnosed at around $12 \%$ to $25 \%$ of the reproductive female population, and in about $80 \%$ of preparations of hysterectomy. ${ }^{1}$ The leiomyomas are monoclonal tumours of myometrial smooth muscle cells that are oestrogen dependent and therefore not seen at prepubertal girls. ${ }^{2}$ There are sporadically described cases in adolescence and most of them shrink in menopause.

\section{Case History}

A 43-year-old patient (G2, P2) was referred by her gynaecologist under a suspected diagnosis of ovarian tumour. Anamnestically, she complained of prolonged and profuse, regular menstrual 
bleeding that led to anaemia. On bimanual examination, a motile, soft uterus was found completely enlarged and pushed forward, and a painfully sensitive tumour mass in the recto-uterine pouch (cavum Douglasi). The ultrasound examination showed a complex tumour mass with dimensions $68 \times 85 \mathrm{~mm}$, with several cystic formations fulfilled with clear fluid, which protruded from the posterior wall of the uterus (Figure 1 and 2). The entire uterus was pushed forward and to the right. The tumour mass was continuous with the rest of the myometrium on the posterior wall of the uterus, indicating uterine origin (Figure 3). The right ovary was displayed separately with a neat morphology and size appropriate for the age. The left ovary was displaced cranially and posteriorly from the tumour mass, but also with neat morphology on ultrasound. Due to the clear ultrasound image of the uterine origin of the tumour, there was no need for MRI preoperatively. Therefore, the diagnosis of leiomyoma with a cystic degeneration was considered preoperatively.

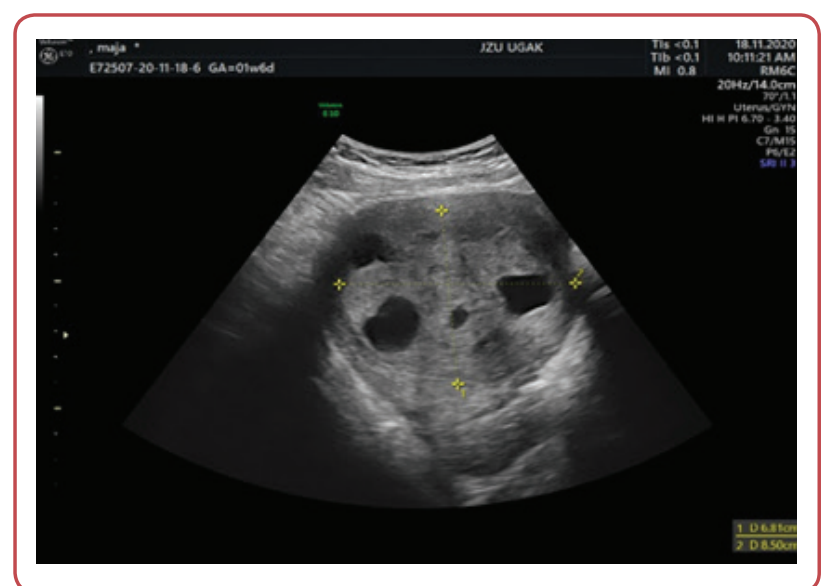

Figure 1: Transabdominal ultrasound - cross sectional imaging of leiomyoma with cystic changes

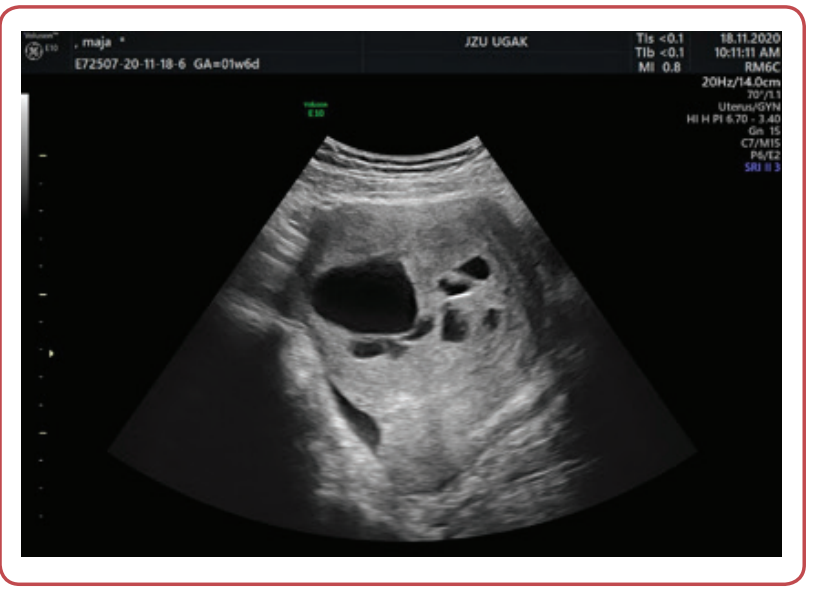

Figure 2: Transabdominal ultrasound - coronal section of a leiomyoma
As part of the preoperative preparation, a fractional exploratory curettage of the body and the cervix of the uterus was performed. The histopathological finding was a functional endometrium under prolonged and exclusively oestrogenic action within the anovulatory cycle.
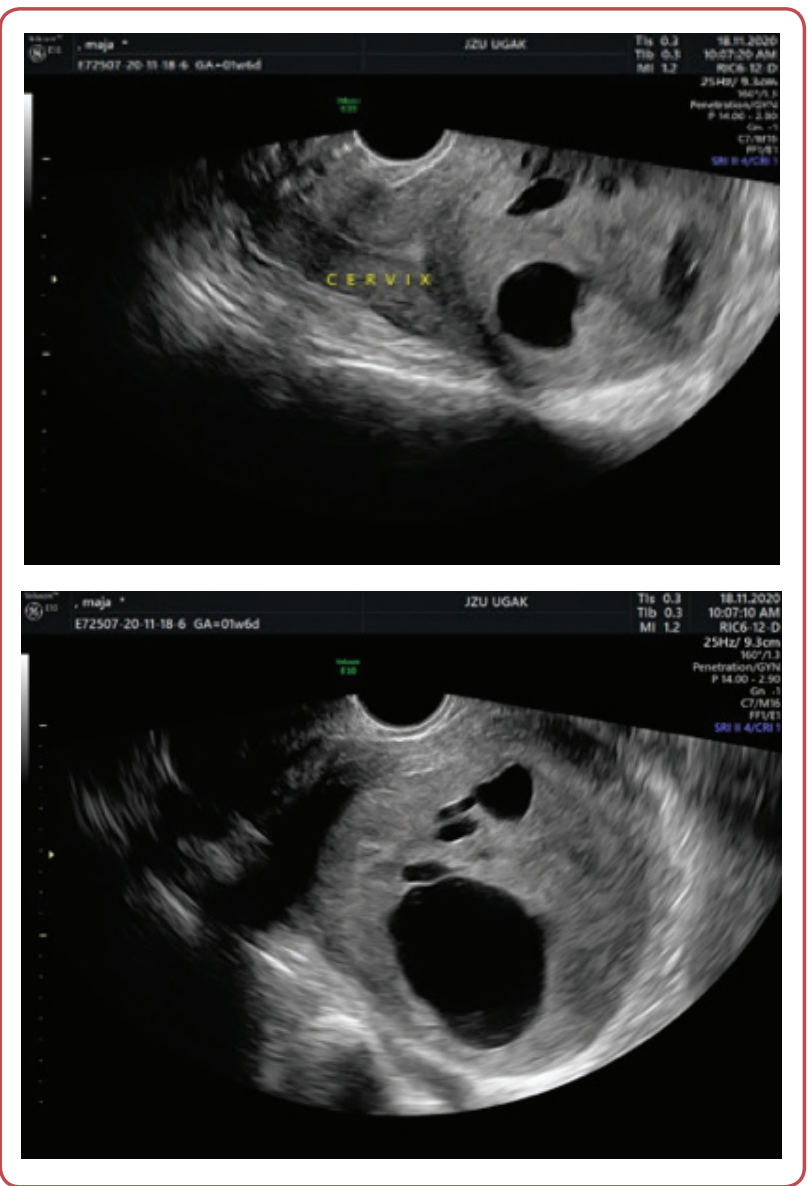

Figure 3: A sagittal, transvaginal ultrasound view of the cervix and the body of the uterus with leiomyoma on the posterior wall

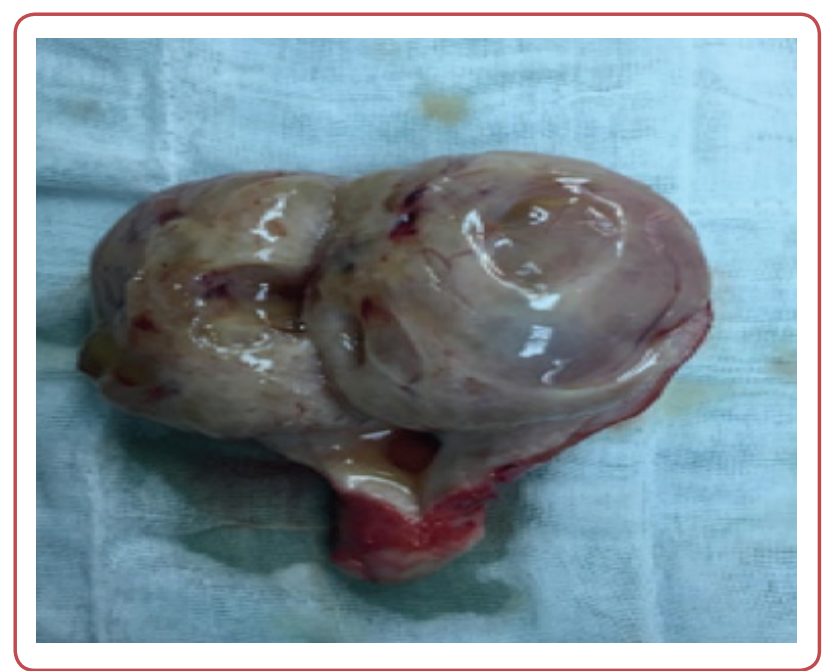

Figure 4: Macroscopic appearance of the specimen from the operation 
The patient underwent a laparotomy and large leiomyoma was found originating from the left part of the posterior uterine wall. Both ovaries were with a neat morphology. Total abdominal hysterectomy with a bilateral salpingectomy was carried out (Figure 4).

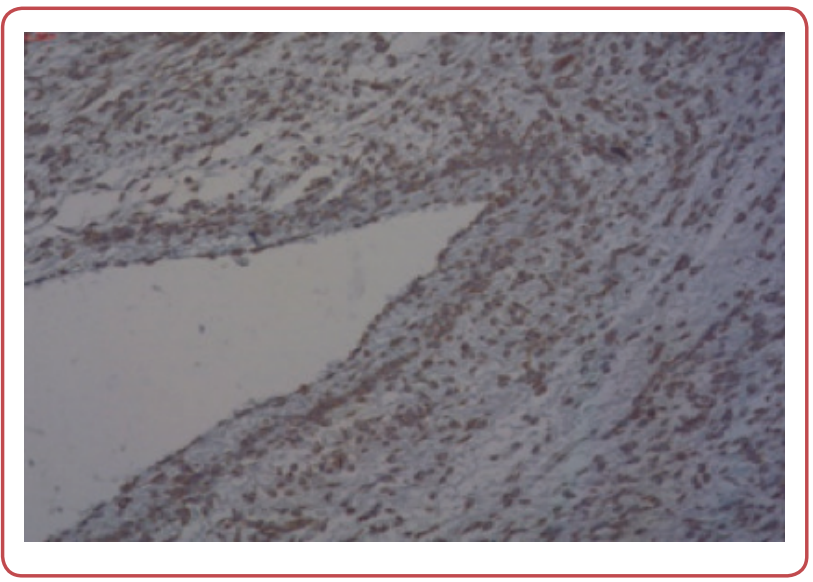

Figure 5: Microscopic specimen after staining with Vimentin

The histopathological analysis showed a leiomyoma with an extensive area of myxoid degeneration and cystic spaces. Immunohistochemical analysis showed that the cystic spaces were coated with smooth muscle actins (SMA) and vimentin positive myocyte cells. Staining for CD34, CD10 and CD31 was negative corresponding to a cystic degenerative leiomyoma (Figure 5).

\section{Discussion}

The leiomyomas are usually diagnosed accidentally during routine gynaecologic examination, or when symptoms appear. The symptoms primarily depend on the location of the myoma (submucosal, intramural or subserosal). Most often it is prolonged and heavy menstrual bleeding and less often pelvic pain or infertility and obstetric complications.

The rapid growth of the myoma ${ }^{3}$ can lead to a relative deficiency of its blood supply and result in various types of degeneration: hyaline (most common, in about $60 \%$ of cases), cystic ( $4 \%$ ), red (3\%), calcified (4\%), myxoid (1 - $3 \%$ ), sarcomatous degeneration $(0.1-0.8 \%)$. The combination of myxoid and cystic degeneration is very rare as only case reports were found in the literature.
Clinically, the leiomyoma degeneration can lead to acute pain, fever, uterine tenderness on palpation, or pressure with an ultrasound probe on the fibroid, elevated leucocytes or even peritoneal signs. ${ }^{4}$ These symptoms, caused by the degeneration, are usually self-limiting and respond well to non-steroidal anti-inflammatory drugs.

The first imaging method of the leiomyoma diagnosis is the ultrasound. At ultrasound, the leiomyoma is presented as hypoechogenic, well-defined round masses, often with shadows. If it is a cellular leiomyoma, then the ultrasound may be isoechoic (making it difficult to differentiate from the surrounding normal myometrium) or hyperechoic. $^{5}$

In the case of cystic degeneration of the myoma, the ultrasound shows a combination of cystic and solid components with irregular shape and variable echogenicity. Due to this, they can be mistaken with a large ovarian simplex cyst, complex endometrial hyperplasia, or postoperative abscess, and therefore, they present a diagnostic challenge. ${ }^{6}$

The first diagnosis to be ruled out in the presence of a large cystic mass with irregular septa and solid nodular parts filling the small pelvis is ovarian malignancy. The exclusion of the ovarian origin is crucial for a differential diagnosis. This can be achieved by showing both ovaries with normal morphology, and on the other hand, showing the continuity of the leiomyoma with the uterine wall. The absence of ascites and other signs of malignancies also support the diagnosis of myoma with cystic degeneration. Low tumour markers can once again confirm the diagnosis pre-operatively.

The differential diagnosis of leiomyoma with cystic degeneration also includes cystic mesothelioma, cystic teratoma, endometriosis, lymphangioma or mesenteric cyst. ${ }^{7}$

In obscure cases, and when a precise diagnosis of complex pelvic mass preoperatively is needed, MRI may be used. The MRI is the most effective method for diagnosing and classification of myomas given the good resolution of the presented soft tissues. 


\section{Conclusion}

Although the myomas are the most common small pelvic tumours in the female population and have a characteristic ultrasound appearance, a diagnostic challenge may occur when degeneration of tumour appears. Ultrasound characteristics of degenerative myomas should always be considered, so as not to replace it with an ovarian mass, especially if it is subserosal and on the loop.

\section{References}

1. Stewart EA, Cookson CL, Gandolfo RA, Schulze-Rath R. Epidemiology of uterine fibroids: a systematic review. BJOG 2017 Sep;124(10):1501-12.

2. Ciavattini A, Di Giuseppe J, Stortoni P, Montik N, Giannubilo SR, Litta P, et al. Uterine fibroids: pathogenesis and interactions with endometrium and endomyometrial junction. Obstet Gynecol Int 2013;2013:173184. doi: 10.1155/2013/173184.

3. Anyanwu M, Gassama K, Kandeh M. Diagnostic dilemma of hyaline cystic degeneration of uterine fibroids. Obstet Gynecol Int J 2019;10(3):202-5.

\section{Acknowledgements}

None.

\section{Conflict of interest}

None.

4. Han SC, Kim MD, Jung DC, Lee M, Lee MS, Park SI, et al. Degeneration of leiomyoma in patients referred for uterine fibroid embolization: incidence, imaging features and clinical characteristics. Yonsei Med J 2013 Jan 1;54(1):215-9.

5. Rashid SQ, Chou YH, Tiu CM. Ultrasonography of uterine leiomyomas. J Med Ultrasound 2016;24(1):3-12.

6. Kaushik C, Prasad A, Singh Y, Baruah BP. Case series: Cystic degeneration in uterine leiomyomas. Indian J Radiol Imaging 2008 Feb;18(1):69-72.

7. Fogata ML, Jain KA. Degenerating cystic uterine fibroid mimics an ovarian cyst in a pregnant patient. J Ultrasound Med 2006 May;25(5):671-4. 